Authors

O. D. Mücke, O. Kuzucu, F. N. C. Wong, E. P. Ippen, F. X. Kaertner, Seth M. Foreman, David J. Jones, LongSheng Ma, John L. Hall, and Jun Ye 


\title{
CPDC9
}

\section{Experimental implementation of optical clockwork without carrier-envelope phase control}

\author{
O. D. Mücke, O. Kuzucu, N. C. Wong, E. P. Ippen, and F. X. Kaertner \\ Department of Electrical Engineering and Computer Science, and Research Laboratory of Electronics, \\ Massachusetts Institute of Technology, 77 Massachusetts Avenue, Cambridge, Massachusetts 02139 \\ Tel:617-253-4186, Fax:617-253-9611, Email: kaertner@mit.edu \\ S. M. Foreman, D. J. Jones, L.-S. Ma, J. L. Hall, and J. Ye \\ IILA, National Institute of Standards and Technology and University of Colorado, \\ Department of Physics, University of Colorado, \\ Boulder, Colorado 80309 \\ Tel: 303-735-3171, Fax: 303-492-5235, Email: Ye@jila.colorado.edu
}

\begin{abstract}
locked Ti:sapphire laser with dominant spectral peaks at 834 and $670 \mathrm{~nm}$.
(92004 Optical Society of America

OCIS codes: (120.3940) Metrology; (320.7090) Ultrafast lasers; (320.7160) Ultrafast technology
\end{abstract}

Abstract: We demonstrate an optical clockwork without carrier-envelope phase control using sum-frequency generation between a CW optical parametric oscillator at $3.39 \mu \mathrm{m}$ and a mode-

\section{Summary:}

The use of phase-stabilized, wide-bandwidth optical frequency combs has revolutionized the field of precision optical frequency metrology and enabled the constnuction of optical atomic clocks [1,2]. Typical setups employ Ti:sapphire lasers producing 10-30-fs pulses with subsequent spectral broadening in microstructure fiber to achieve octave spanning spectra which are required for f-to-2f self-referencing [3]. More recent laser systems that directly produce octave-spanning spectra for frequency comb stabilization have been demonstrated [4]. Our work here demonstrates optical clockwork without the need for carrier-envelope phase control, simplifying the implementation of the clockwork and avoiding the necessity of an octave-spanning spectrum.

Recently, A. Baltuška et al. [5] have shown that the frequency comb generated by a pulsed optical parametric oscillator (OPO) can eliminate carrier-envelope frequency shifts. This has been further investigated using difference-frequency generation [6]. Here, we implement an optical clockwork without the need for carrier-envelope phase control. Our scheme is based on sum-frequency generation (SFG) between a CW OPO at $3.39 \mu \mathrm{m}$ and a specially designed mode-locked Ti:sapphire laser with two dominant spectral peaks near $834 \mathrm{~nm}$ and $670 \mathrm{~nm}$. The setup is shown in Figure 1.

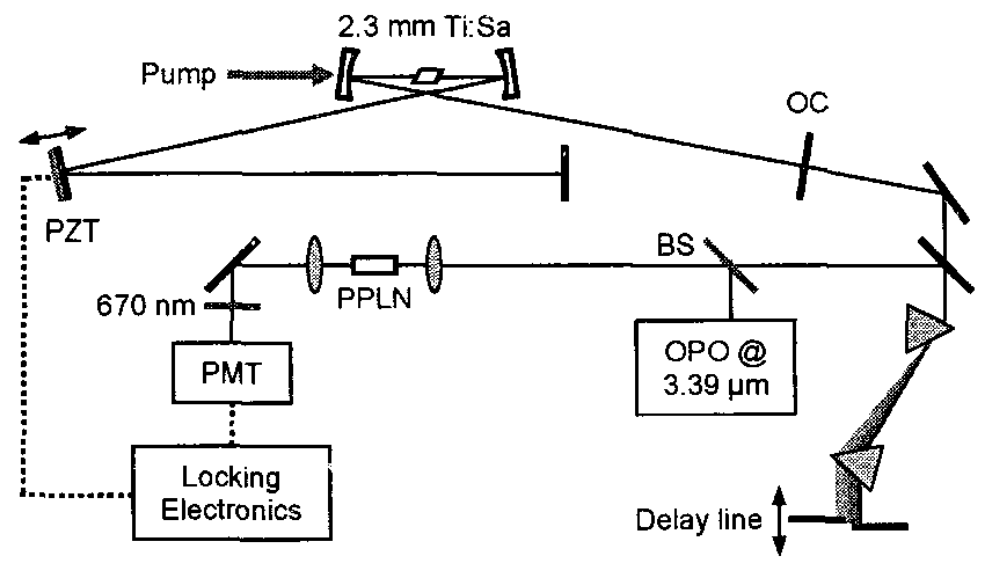

Figure 1: Setup for SFG-based optical heterodyne beat for the implementation of an optical clock without carrier-envelope phase control. Note $3.39 \mu \mathrm{m}+834 \mathrm{~nm} \rightarrow 670 \mathrm{~nm}$. All intracavity mirrors are double-chirped mirrors. 


\section{CPDC9}

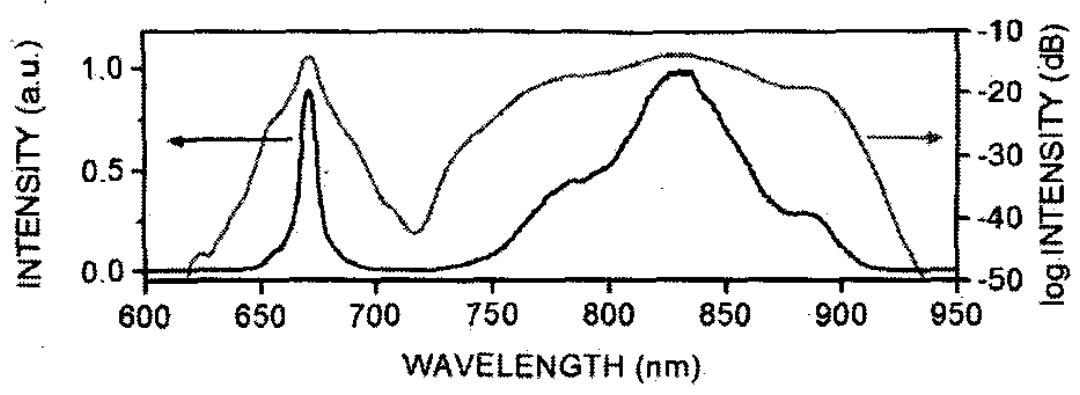

Figure 2: Output spectrum of the Tissapphire laser. The wings of the spectrum at $670 \mathrm{~nm}$ and $834 \mathrm{~nm}$ are enhanced due to the roll-off of the output coupler. The amplitude of the short-wavelength peak is very sensitive to intracavity dispersion.

The spectrum of the Tissapphire laser as shown in Fig. 2 is generated using a narrowband output coupler. A transmission of about $1 \%$ occurs at the center of the output coupler. The coupler rolls off strongly at the designated wavelengths of $690 \mathrm{~nm}$ and $866 \mathrm{~nm}$. At these wavelengths the transmission increases to $5 \%$ leading to two pronounced spectral peaks in the output spectrum. The Ti:sapphire laser runs at a repetition rate of $78 \mathrm{MHz}$.with an average output power of typically $150 \mathrm{~mW}$. Using a $40 \mathrm{~mm}$ focal length calcium fluoride lens, the $28 \mathrm{~mW}$ of output power of the Ti:sapphire laser near $834 \mathrm{~nm}$ and the $16 \mathrm{~mW} \mathrm{CW}$ OPO output at $3.39 \mu \mathrm{m}$ are focused into a $5 \mathrm{~mm}$ long periodically-poled Lithium Niobate (PPLN) crystal heated to $130^{\circ} \mathrm{C}$ with a quasi-phase matching period of 16.9 $\mu \mathrm{m}$. The corresponding phase matching bandwidth of this PPLN crystal is around $1 \mathrm{THz}$. The pump laser of the doubly resonant CW OPO is a Nd:YAG laser and the OPO cavity is frequency locked to the pump laser. The idler output is used for SFG.

The resultant SFG comb and the original Ti:sapphire comb at $670 \mathrm{~nm}$ are temporally and spatially overlapped using a prism-based delay line (Fig. 1). A heterodyne beat signal between the two combs is detected by a photomultiplier with a signal-to-noise ratio of $\sim 25 \mathrm{~dB}$ in a $30 \mathrm{kHz}$ resolution bandwidth (Fig. 3(a)). Please note that the final beat signal results from the coherent superposition of many corresponding comb lines. Importantly, frequency variation of this beat signal depends only on the laser repetition frequency but not on the carrier-envelope frequency. By phase locking this beat signal to a radio-frequency reference, we therefore lock the Ti:sapphire repetition frequency directly to the OPO optical frequency (Fig. 3(b)). The drift and small sinusoidal fluctuations in the stabilized repetition frequency are due to those of the OPO pump laser. The OPO's pump laser will be phase locked to our iodine-stabilized Nd:YAG laser [2], thus establishing an optical atomic clock independent of the carrier-envelope frequency.
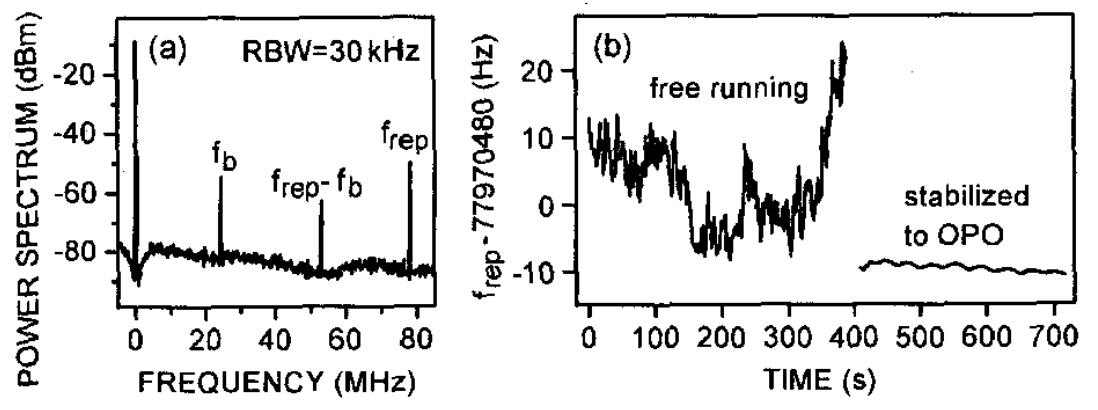

Figure 3: (a) Heterodyne beat between the SFG comb and the original comb. (b) Frequency counting of the laser repetition frequency $f_{\text {rep }}$ under free-running conditions and while stabilized to the CW OPO.

\section{References}

1. S. A. Diddams, et al, Science 293, 825-828 (2001).

2. J. Ye et al., Phys. Rev. Lett. 87, 270801-1-4 (2001).

3. D. J. Jones et al., Science 288, 635-639 (2000).

4. R. Ell et al., Opt. Lett. 26, 373-375 (2001); T. M. Fortier et al., Opt. Lett. 28, 2198-2200 (2003).

5. A. Baltuška et al., Phys. Rev. Lett. 88, 133901-1-4 (2002).

6. M. Zimmermann et al, Opt. Lett. 29, 310-312 (2004). 